\title{
DE NUEVO SOBRE CONSTITUCIÓN Y DISCAPACIDAD \\ AGAIN ON CONSTITUTION AND DISABILITY
}

\author{
Rafael de Asís*
}

RESUMEN: El trabajo comienza con el análisis de la propuesta de reforma del artículo 49 de la Constitución del año 2018 y del dictamen que al respecto realizó el Consejo de Estado. A partir de ahí, se expone el marco de referencia con el que debe realizarse una reforma del tratamiento de la discapacidad en la Constitución ajustado al discurso de los derechos humanos.

ABSTRACT: The work begins with the analysis of the proposed reform in the year 2018 of article 49 of the Constitution and the opinion that the State Council made in this regard. From there, the frame of reference with which a reform of the treatment of disability in the Constitution adjusted to the discourse of human rights should be made is exposed.

PALABRAS ClAVE: discapacidad, derechos humanos, Constitución, reforma.

KEYWORDS: disability, human rights, Constitution, reform.

Fecha de recepción: 06/10/2019

Fecha de aceptación: 13/12/2019

doi: https://doi.org/10.20318/universitas.2020.5137

* Catedrático de Filosofía del Derecho de la Universidad Carlos III de Madrid. Email: rarfid@inst.uc3m.es 
Como es sabido, el diciembre de 2018, el Consejo de Ministros aprobó un Anteproyecto de Ley para la reforma del artículo 49 de nuestra Constitución. El texto propuesto decía ${ }^{1}$ :

"1. Las personas con discapacidad son titulares de los derechos $y$ deberes previstos en este Título en condiciones de libertad e igualdad real y efectiva, sin que pueda producirse discriminación. 2. Los poderes públicos realizarán las políticas necesarias para garantizar la plena autonomía personal e inclusión social de las personas con discapacidad. Estas políticas respetarán su libertad de elección y preferencias, y serán adoptadas con la participación de las organizaciones representativas de personas con discapacidad. Se atenderán particularmente las necesidades específicas de las mujeres y niñas con discapacidad. 3. Se regulará la protección reforzada de las personas con discapacidad para el pleno ejercicio de sus derechos y deberes. 4. Las personas con discapacidad gozan de la protección prevista en los acuerdos internacionales que velan por sus derechos".

Al parecer, existía en aquel momento un cierto consenso entre las fuerzas parlamentarias en relación con esta reforma, que podía hacerse por vía del artículo 167 de la Constitución y siguiendo las previsiones de los artículos 146 del Reglamento del Congreso y 154 a 157 del Reglamento del Senado.

La coyuntura política española provocó que no pudieran seguirse los trámites preceptivos y habrá que esperar a que los avatares políticos se resuelvan para ver si este trabajo se retoma.

En todo caso, en las páginas siguientes voy a referirme al texto del anteproyecto y a la oportunidad de acometer la reforma de la Constitución en materia de discapacidad.

\section{1.- LA PROPUESTA DE REFORMA DEL ARTÍCULO 49 DE LA CONSTITUCIÓN}

En la exposición de motivos del anteproyecto, se justifica la reforma aludiendo, en primer lugar, a la existencia de una demanda, por parte del colectivo de personas con discapacidad y sus representantes, de acomodar el artículo 49 a la realidad social y con ello sentar las bases de una acción pública más vigorosa y eficaz. Se afirma que el artículo 49 precisa una actualización en su terminología y en el enfoque de la discapacidad, al estar basado en el modelo médico-rehabilitador y no en el modelo social. Se busca así también superar un enfoque excesivamente paternalista de la discapacidad.

\footnotetext{
1 Puede consultarse en:

https://www.mpr.gob.es/prencom/notas/Documents/071218_Art49Consti.pdf
} 
En resumidas cuentas, se dice que se pretende que el precepto vuelva a ser una referencia en la protección de los derechos de las personas con discapacidad.

La nueva redacción modifica en primer lugar la terminología, refiriéndose ahora a las personas con discapacidad. De esta manera, se actualiza el lenguaje de una forma que refleja los propios valores de la Constitución y la dignidad inherente a este colectivo.

Igualmente, se subrayan sus derechos y deberes y se fija la autonomía personal y la inclusión social como objetivos de las políticas públicas. Además se afirma que estas políticas públicas deberán respetar la libertad de elección de las personas con discapacidad y ser elaboradas junto a las organizaciones representativas de personas con discapacidad.

La reforma se refiere explícitamente a las necesidades específicas de las mujeres y niñas con discapacidad.

Por otro lado, se incorpora, apoyándose en la Sentencia del Tribunal Constitucional 208/2013 de 16 de diciembre que se refiere a la protección especial de las personas con discapacidad, un apartado sobre protección reforzada de las personas con discapacidad para el pleno ejercicio de sus derechos.

Y por último, la reforma realiza una mención general a los acuerdos internacionales sobre derechos de las personas con discapacidad.

El 28 de febrero de 2019, el Consejo de Estado emitió un dictamen sobre el anteproyecto de reforma en el que se apuntaban algunas consideraciones que, necesariamente, hay que tener en cuenta.

Es de subrayar que el dictamen comienza cuestionando en cierto sentido la necesidad de reformar el artículo 49, al afirmar que en su redacción actual puede interpretarse, en virtud de los artículos 10,2 y 96,1 de la Constitución, conforme a la Convención sobre los Derechos de las Personas con Discapacidad.

Esta consideración del Consejo de Estado, había sido ya adelantada por alguno de sus miembros. En concreto, Miguel Rodríguez-Piñeiro, había ya comentado que "no es correcta la frecuente interpretación del art. 49 CE que ve en él, al margen de la obsoleta terminología que utiliza, una consagración de una visión meramente reparadora o médica del tratamiento de la discapacidad, ya que en su texto se incluye una protección jurídica desde un enfoque de derechos que puede considerarse como una justificación implícita de una consideración «social» de la discapacidad, luego reconocida por la normativa internacional de derechos humanos y por la legislación interna que desarrolla el precepto constitucional y esa normativa internacional"2.

${ }^{2}$ Rodríguez-Piñeiro, M., "Artículo 49", en Rodriguez-Piñeiro, M. y Casas, M.E., Comentarios a la Constitución española, BOE-Wolters Kluwer, Madrid 2018, p. 1.405 . 
Por otro lado, y en relación con este tema, llama la atención que el Comité sobre los Derechos de las Personas con Discapacidad de Naciones Unidas, en su informe sobre España publicado en abril de 2019, no haya hecho mención alguna al contenido de este artículo, lo que para algunos podría apoyar la tesis antes expuesta ${ }^{3}$.

En este segundo informe sobre España (el primero se publicó en octubre de 2011), el Comité felicita al Estado por la aprobación de la Ley Orgánica 2/2018, que modifica la Ley Orgánica 5/1985 del Régimen Electoral General, y de la Ley Orgánica 1/2017, que garantiza la no exclusión de las personas con discapacidad del tribunal del jurado. También felicita al Estado por la aprobación o modificación de otras normas, si bien, luego a lo largo del documento mantiene observaciones críticas al respecto. Se trata de la Ley de derechos de las personas con discapacidad y de su inclusión social (Real Decreto 1/2013), de la Ley Orgánica 13/2015 (de modificación de la Ley de Enjuiciamiento Criminal), de la Ley Orgánica 1/2015 (modifica el Código Penal) o de la reforma de la Ley 9/2017, de Contratos del Sector Público. Se congratula el Comité por la existencia del Plan de Acción para la aplicación de la Estrategia Española sobre Discapacidad 2012-2020 a nivel nacional y por la aprobación de otros planes elaborados por las distintas comunidades autónomas.

Dos son los asuntos que destaca el Comité como preocupantes por encima de cualquiera otros: las esterilizaciones forzosas y la cuestión educativa. Así, en el parágrafo 64 del documento puede leerse: "El Comité destaca la importancia de todas las recomendaciones que figuran en las presentes observaciones finales. En particular, el Comité señala a la atención del Estado parte las recomendaciones relativas al artículo 17 (Protección de la integridad personal) y 24 (Educación inclusiva) de la Convención, respecto de las cuales deben adoptarse medidas urgentes" (esto último en relación con el cumplimiento del informe de su investigación, CRPD/C/20/3).

Pero a pesar de ser un informe más prolijo que el primero, el Comité no menciona el artículo 49 de la Constitución ${ }^{4}$.

\footnotetext{
${ }^{3}$ El informe está disponible en:

https://tbinternet.ohchr.org/_layouts/15/treatybodyexternal/Download.aspx?symb olno $=$ CRPD $\% 2 \mathrm{fC} \% 2 \mathrm{fESP} \% 2 \mathrm{fCO} \% 2 \mathrm{f} 2-3 \&$ Lang $=$ en

${ }^{4}$ En efecto, en el segundo informe hay observaciones y recomendaciones de once artículos en relación con los cuales el primer informe no se pronunciaba. Se trata de los artículos 13 (acceso a la justicia), 15 (protección contra la tortura y otros tratos o penas crueles, inhumanos o degradantes), 16 (protección contra la explotación, la violencia y el abuso), 18 (libertad de desplazamiento y nacionalidad), 21 (libertad de expresión y de opinión y acceso a la información), 22 (respeto de la privacidad), 23 (respeto del hogar y de la familia), 25 (salud), 30 (participación en la vida cultural, las actividades recreativas, el esparcimiento y el deporte), 32 (cooperación internacional) y 33 (aplicación y seguimiento nacionales). Si se tiene en cuenta que el informe se centra sobre 33 artículos de la Convención, es una cifra importante, y lo es aún más si tenemos en cuenta que ni
} 
Volviendo al dictamen sobre el anteproyecto de reforma del artículo 49, el Consejo de Estado realiza cuatro consideraciones que se proyectan sobre cada uno de los apartados del artículo en su nueva redacción.

En primer lugar, el dictamen se refiere a la proclamación de que las personas con discapacidad son titulares de los derechos y deberes previstos en el Título I de la Constitución. Al Consejo de Estado no le parece pertinente porque considera que es una afirmación innecesaria por evidente, pero también porque no es apropiada teniendo en cuenta que estamos en el capítulo de los principios rectores (que poseen un grado normativo menor). En este punto el Consejo de Estado se agarra a la distinción entre titularidad y ejercicio de los derechos.

Respecto al apartado segundo, cuestiona la alusión a las organizaciones representativas de las personas con discapacidad en la adopción de las políticas sobre la discapacidad. Al respecto, considera que no se trata de una redacción habitual, que esa participación debe ser objeto de regulación legal, y que su elevación a rango constitucional puede tener efectos perturbadores.

En relación con el apartado tercero, cuestiona la referencia a la protección reforzada. Y lo hace afirmando que se trata de un concepto jurídico indeterminado cuya interpretación puede distorsionar la finalidad que se persigue, y señalando también que la protección que cabe es la que establece el art. 53,3 de la Constitución que, para este caso, no puede entenderse como directa e inmediata.

Al hilo de esta consideración, el Consejo de Estado señala la conveniencia de llevar esta cuestión de la protección al mismo lugar en el que el artículo se refiere a la titularidad.

Respecto al apartado cuarto, el Consejo de Estado considera que la alusión a los Tratados internacionales resulta también reiterativa.

En virtud de todo lo anterior, el Consejo de Estado propone introducir los siguientes cambios:

Refundir los incisos 1 y 3 : Las personas con discapacidad ejercen los derechos y deberes previstos en este Título en condiciones de libertad e igualdad real y efectiva. Se regulará la protección especial que sea necesaria para dicho ejercicio.

el primer informe ni el segundo contienen observaciones sobre el artículo 20 (movilidad personal) ni sobre el 26 (habilitación y rehabilitación). Por otro lado, si cuantificamos las observaciones y recomendaciones por artículos, el segundo informe incorpora 33 nuevas observaciones y recomendaciones. Sin embargo, en realidad no son realmente nuevos problemas sino, en muchos casos, asuntos que ya estaban presentes pero que ahora se vinculan a nuevos artículos. Sobre el informe vid. De Asís, R., "El segundo informe sobre cumplimiento por parte de España de la Convención sobre los derechos de las personas con discapacidad", en Papeles El Tiempo de los Derechos, n. 16, 2019. 
Completar la referencia a la participación de las organizaciones profesionales -contenida en el apartado 2 del artículo 49- con la remisión a lo que disponga una norma con rango de ley.

Eliminar el apartado 4 del artículo 49 (sin la mención de los acuerdos internacionales queda vacío de contenido).

Aunque algunas de las consideraciones pueden ser, en línea de principio, adecuadas en términos de técnica normativa, no hay que olvidar que estamos ante un asunto de protección de derechos y de lucha contra la discriminación.

Las consideraciones sobre el primer apartado parecen el algún momento incoherentes. Se afirma que es evidente que las personas con discapacidad tienen los mismos derechos que cualquier otra persona, pero también que no es apropiado decirlo en el capítulo III... Y así, propone un cambio basado en la distinción entre titularidad y ejercicio. Pero no olvidemos que esa diferencia ha tenido grandes (y graves) consecuencias en el disfrute de los derechos de las personas con discapacidad.

En relación con el segundo apartado, es cierto que puede sorprender y parecer inadecuada la referencia a las organizaciones de la discapacidad. Sin embargo, no estoy seguro que el dictamen entienda su papel y su historia. Sobre todo porque cuando se refiere al texto que se sugiere en sustitución del proyecto se habla de organizaciones profesionales.

Respecto al apartado 3, lo que dice el Consejo de Estado pone de manifiesto la regulación cuestionable de los derechos de las personas con discapacidad, fruto de una comprensión también cuestionable.

Los derechos de las personas con discapacidad son los mismos que los de cualquier otra persona y pueden ser protegidos por las mismas vías (directa e inmediata). No son principios rectores a pesar de que se aluda a las personas con discapacidad en el artículo 49. Además, dada la situación de discriminación en la que se encuentran las personas con discapacidad hablar de protección reforzada (nunca especial), puede tener sentido.

Nada que decir sobre las consideraciones del apartado 4. En mi opinión tiene razón el Consejo de Estado. No obstante, esa alusión a los textos internacionales se explica por el escaso éxito que estos textos tienen entre los operadores jurídicos y en la sociedad en general. Seguimos sin tomarnos en serio el Derecho internacional ${ }^{5}$.

Vaya por delante que, en lo referente a los derechos humanos, todo es insuficiente. Pero dicho esto, el anteproyecto de reforma del artículo 49 era un paso adelante.

Es cierto que muchas de las cosas estaban ya en otros preceptos o habían sido adelantadas por el Tribunal Constitucional,

\footnotetext{
${ }^{5}$ Y ello vuelve a poner sobre la mesa la relevancia de la formación y educación en derechos humanos... Mientras esto no ocurra, tal vez haya que seguir aludiendo a lo obvio en las normas.
} 
pero aparecer explícitamente en relación con las personas con discapacidad no estaba de más. Es el caso de la prohibición de la discriminación o de la alusión a los tratados internacionales. Esto último puede entenderse, además, como un reconocimiento a la Convención sobre los derechos de las personas con discapacidad, principal motor de la nueva visión de la discapacidad.

La alusión al movimiento asociativo, siempre y cuando se interpretara en términos de pluralidad, parece adecuada $y$, al igual que en el caso de la Convención, es un reconocimiento a la labor de todas las asociaciones y entidades.

Ahora bien la propuesta de reforma podría haber sido mejor. En el punto siguiente me referiré a cuales deben los referentes de una posible reforma de la Constitución en lo referente al tratamiento de la discapacidad.

\section{2.- BASES PARA UNA POSIBLE REFORMA DEL TRATAMIENTO DE LA DISCAPACIDAD EN LA CONSTITUCIÓN}

Hace varios años escribía en la Revista Derechos y Libertades, del Instituto de Derechos Humanos Bartolomé de las Casas, un artículo titulado "Discapacidad y Constitución"6, en el que señalaba que para la satisfacción de los derechos de las personas con discapacidad, no bastaba con interpretar los derechos (en virtud del mandato del artículo 10,2 de la Constitución) de conformidad con Tratados Internacionales como la Convención sobre los derechos de las personas con discapacidad7. Era necesario reformar la Constitución y adaptarla al modelo social de la discapacidad. Pues bien, esa necesidad sigue estando presente y es cada vez más necesaria.

Como es sabido, el modelo social de la discapacidad se caracteriza, en términos generales por mantener una idea de discapacidad que combina rasgos de las personas con la situación en la que se encuentran 0 , si se prefiere, rasgos y barreras $^{8}$. Así, La Convención Internacional sobre los Derechos de las Personas con Discapacidad señala en su art. 1: "Las personas con discapacidad incluyen a aquellas que tengan deficiencias físicas, mentales, intelectuales o sensoriales a largo plazo que, al interactuar con diversas barreras, puedan impedir su participación plena y efectiva en la sociedad, en igualdad de condiciones con las demás". Pero además, el modelo social supone la incorporación definitiva de la discapacidad al discurso de los derechos.

\footnotetext{
${ }^{6}$ De Asís, R., "Discapacidad y Constitución", en Derechos y Libertades, n. 29, 2013, pp. 39 y ss.

7 Vid. la Sentencia del Tribunal Constitucional 3/2018, de 22 de enero.

8 Para una caracterización más completa vid. Palacios, A., El modelo social de la discapacidad, Colección CERMI, Madrid 2008, pp. 103 y ss.
} 
Pues bien, una reforma de la Constitución en esta materia requiere partir de dos presupuestos: (i) las personas con discapacidad tienen los mismos derechos que cualquier otra y, (ii) sus derechos no son solo derechos sociales.

A partir de ahí, la reforma de la Constitución en materia de discapacidad afecta, necesariamente, a dos artículos: el 14 y el 49.

En lo que respecta al artículo 14, la entrada de la discapacidad tiene cabida en la referencia final del precepto al hablar de "cualquier otra condición o circunstancia personal o social", y así lo reconoció nuestro Tribunal Constitucional en su Sentencia 269/94 de 3 de octubre. Además, la prohibición de discriminación por razón de discapacidad forma parte de nuestro Ordenamiento en virtud de diversas normas de la Unión Europea, entre las que destacan el artículo 13 del Tratado de Amsterdam, la Directiva 2000/78/CE y el artículo 21 de la Carta de Derechos Fundamentales. Sin embargo, considero preciso introducir expresamente el término discapacidad entre las prohibiciones de discriminación.

Y respecto al artículo 49, podría pensarse que es posible reconducirlo a lo establecido en la Convención vía interpretativa, tal y como se ha apuntado. Una interpretación del artículo 49, conforme a la Convención, implicaría, al menos: (i) incluir a la accesibilidad entre las políticas de previsión; (ii) entender que el tratamiento de la discapacidad no es sólo una cuestión relacionada con políticas sanitarias y de seguridad social sino principalmente una cuestión de derechos humanos (iii) considerar que las políticas de rehabilitación tienen como principal destinatario a la sociedad en su conjunto y se relacionan estrechamente con la accesibilidad; (iv) entender que la integración implica inclusión en todas las parcelas de la vida social.

Pero ya he señalado como este camino puede no ser satisfactorio. Además, no cabe duda de que hay que suprimir el término disminuido $y$ sustituirlo por otro no discriminatorio ni estigmatizante, y acorde con el discurso de los derechos humanos. Seguramente, en este punto, en consonancia con la normativa internacional, el término mejor sea el de persona con discapacidad (aunque se proponen otros como persona con diversidad funcional).

Pero también debería suprimirse del artículo 49 el término integración y sustituirlo por el de inclusión, mucho más acorde con el discurso de los derechos humanos. Y ya de paso, aludir a políticas de remoción de barreras y de participación.

Además, podría trasladarse el artículo 49 a la sección $1^{\text {a }}$ del Capítulo $2^{\circ}$ del Título $1^{\circ}$ de la Constitución, pero esto no debe justificarse solo desde el discurso (que comparto) de la "fundamentalidad" de los derechos económicos, sociales y culturales (porque los derechos de las personas con discapacidad no son sólo derechos económicos, sociales y culturales). En todo caso, puede tener más sentido trasladarlo a la sección $2^{\mathrm{a}}$ ya que, como he señalado, las personas con discapacidad gozan de todos los derechos constitucionales, y su mención en la sección $1^{a}$ puede mantener el 
discurso de lo especial que normalmente les acompaña. Por último, también podría dejarse donde está, siempre y cuando se modificará el artículo 53 y con ello la distinción entre derechos fundamentales y constitucionales.

Trasladar lo dispuesto en el artículo 49 a la sección $1^{a}$ o a la segunda del Capítulo segundo del Título 10, adquiriría pleno sentido si, además, se incorporaran al texto, otras exigencias en forma de derechos, presentes en la Convención sobre los derechos de las personas con discapacidad.

Estas exigencias serían las de la accesibilidad universal, los ajustes razonables, la igual capacidad jurídica y los apoyos, la vida independiente y la asistencia personal y el reconocimiento del uso de la lengua de signos. Se trata de los principales aspectos en materia de derechos y que poseen cierta singularidad en relación con las personas con discapacidad.

Suele ser común afirmar que la Convención es un Tratado de derechos humanos que se caracteriza por no reconocer nuevos derechos. Se afirma así que lo que hace la Convención es establecer que las personas con discapacidad poseen los mismos derechos que cualquier otra persona9. La Convención es así un texto que lucha contra la discriminación en la que se encuentran estas personas y que se traduce en la ausencia de satisfacción de sus derechos humanos.

Pues bien, a pesar de que ese pueda ser el sentido general de la Convención, es posible no obstante afirmar que este Tratado sí que reconoce unos nuevos derechos para las personas con discapacidad. Se trata, en algunos casos, de derechos que han sido tradicionalmente negados a este colectivo, y en otros, de auténticos nuevos derechos que tienen su origen en demandas específicas.

En este sentido, es posible referirse a cuatro nuevos derechos que de manera explícita o de forma implícita están recogidos en la Convención. En concreto se trata del derecho a una igual capacidad jurídica, del derecho a la accesibilidad universal, del derecho a vivir de forma independiente y a ser incluido en la comunidad, y del derecho al uso de la lengua de signos.

El derecho a una igual capacidad jurídica aparece en el artículo 12 de la Convención en cuyo punto segundo se afirma: "Los Estados Partes reconocerán que las personas con discapacidad tienen capacidad jurídica en igualdad de condiciones con las demás en todos los aspectos de la vida". Se trata de un derecho en el que se integra también el derecho a recibir los apoyos necesarios para el ejercicio de dicha capacidad ${ }^{10}$, que aparece recogido en el punto 3 de ese mismo

\footnotetext{
9 Vid. Palacios, A., El modelo social de la discapacidad, cit., pp. 257 y ss.

10 Vid. Bariffi, F., "Capacidad jurídica y capacidad de obrar de las personas con discapacidad a la luz de la Convención de la ONU", en Pérez Bueno, L.C. (Dir.), Hacia un Derecho de la Discapacidad. Estudios en homenaje al Profesor Rafael de Lorenzo, Thomson Reuters Aranzadi, Pamplona, 2009, pp. 353 y ss.
} 
artículo en el que puede leerse: "Los Estados Partes adoptarán las medidas pertinentes para proporcionar acceso a las personas con discapacidad al apoyo que puedan necesitar en el ejercicio de su capacidad jurídica"11.

El derecho a vivir de forma independiente y a ser incluido en la comunidad aparece explícitamente reconocido en el artículo 19 de la Convención ${ }^{12}$. El reconocimiento de este derecho supone, en virtud del apartado a) de ese precepto, la obligación de los Estados de asegurar que las personas con discapacidad "tengan la oportunidad de elegir su lugar de residencia y dónde y con quién vivir, en igualdad de condiciones con las demás, y no se vean obligadas a vivir con arreglo a un sistema de vida específico". Y este derecho implica también el derecho a "la asistencia personal que sea necesaria para facilitar su existencia y su inclusión en la comunidad y para evitar su aislamiento o separación de ésta" (apartado b) del artículo 19).

El derecho al uso de la lengua de signos, que en realidad implica uso, reconocimiento y protección ${ }^{13}$, aparece en diversos artículos de la Convención, si bien es en el artículo 30, dedicado a la participación en la vida cultural, las actividades recreativas, el esparcimiento y el deporte, donde, en su apartado 4, se señala: "Las personas con discapacidad tendrán derecho, en igualdad de condiciones con las demás, al reconocimiento y el apoyo de su identidad cultural y lingüística específica, incluidas la lengua de señas y la cultura de los sordos".

He dejado para el final la accesibilidad universal. Y lo he hecho porque me parece el derecho más importante que, además, se proyecta mucho más allá de la discapacidad.

La accesibilidad es una idea esencial en cualquier contexto ${ }^{14}$. El artículo 9 de la Convención sobre los derechos de las personas con discapacidad se refiere a la accesibilidad universal afirmando: "A fin de que las personas con discapacidad puedan vivir en forma independiente y participar plenamente en todos los aspectos de la vida, los Estados Partes adoptarán medidas pertinentes para asegurar el acceso de las personas con discapacidad, en igualdad de condiciones con las demás, al entorno físico, el transporte, la información y las comunicaciones, incluidos los sistemas y las tecnologías de la información y las comunicaciones, y a otros

11 Sobre el tema vid. Cuenca, P., "La capacidad jurídica de las personas con discapacidad: el art. 12 de la Convención de la ONU y su impacto en el Ordenamiento jurídico español", en Derechos y Libertades, n. 24, 2011, pp. 221 y ss.

12 Vid. al respecto Barranco Avilés, M.C y De Asís, R., "El derecho a la promoción de la autonomía personal y atención a las personas en situación de dependencia", en Barranco, M.C. (coord.), Situaciones de dependencia, Discapacidad y Derechos, Dykinson, Madrid 2011, pp. 107 y ss.

13 Vid. De Asís, R., "Sobre el derecho al conocimiento y aprendizaje de la lengua de signos", en Cuenca, P. (ed.), Estudios sobre los derechos de las personas sordas, Dykinson, Madrid 2012, pp. 99 y ss.

14 Vid. Kalbag, L., Accessibility for Everyone, A Book Apart, New York 2017. 
servicios e instalaciones abiertos al público o de uso público, tanto en zonas urbanas como rurales".

Esta exigencia adquiere sentido en relación con el diseño universal y los ajustes razonables. El diseño universal es definido en el artículo 2 de la Convención como "el diseño de productos, entornos, programas y servicios que puedan utilizar todas las personas, en la mayor medida posible, sin necesidad de adaptación ni diseño especializado". Y el mismo precepto define los ajustes razonables como: "las modificaciones y adaptaciones necesarias y adecuadas que no impongan una carga desproporcionada o indebida, cuando se requieran en un caso particular, para garantizar a las personas con discapacidad el goce o ejercicio, en igualdad de condiciones con las demás, de todos los derechos humanos y libertades fundamentales".

La lectura del artículo 9 de la Convención parece relacionar la accesibilidad con una serie de ámbitos: entorno físico, el transporte, la información y las comunicaciones, y otros servicios e instalaciones abiertos al público o de uso público. Y es muy habitual concebir así la accesibilidad.

No obstante, el artículo hace mención a otros tres grandes derechos que sirven para justificar la accesibilidad y que permiten ampliar enormemente su proyección. Se trata de la vida independiente, de la participación en la vida social y de la igualdad de oportunidades.

Es posible hablar así de un sentido restringido de accesibilidad y de un sentido amplio. El sentido restringido de la accesibilidad, que se proyecta sobre "productos, entornos, programas y servicios", supone "el acceso de las personas con discapacidad, en igualdad de condiciones con las demás, al entorno físico, el transporte, la información y las comunicaciones, incluidos los sistemas y las tecnologías de la información y las comunicaciones, y a otros servicios e instalaciones abiertos al público o de uso público, tanto en zonas urbanas como rurales". El sentido amplio de la accesibilidad supone el acceso a todos los bienes y derechos y se fundamenta en la vida independiente, en la participación en la vida social y en la igualdad de oportunidades; conecta con la idea de capacidad, y subraya su dimensión como posibilidad o, si se quiere como derecho a tener derechos.

La accesibilidad en sentido restringido se manifiesta principalmente como principio y como derecho subjetivo mientras que la accesibilidad en sentido amplio, en lo referido a derechos y bienes, lo hace como parte del contenido esencial del derecho en cuestión o como derecho a no ser discriminado.

Por este motivo, también tendría sentido dedicar un precepto constitucional a la accesibilidad, aludir a esta al referirse a la no discriminación o al contenido esencial de los derechos.

$Y$ desde una perspectiva que excede el campo de la discapacidad pero en estrecha relación con ella, parece importante 
dedicar algún precepto constitucional a lo que podríamos denominar como atención al desarrollo y a una de sus proyecciones: la atención temprana ${ }^{15}$. La atención al desarrollo puede ser entendida como un derecho de aquellos/as menores que presentan algún tipo de barrera al desarrollo o están en situación de riesgo de tenerla. La eliminación de esas barreras es fundamental en los primeros años de la vida de las personas. Así, en relación con los niños y las niñas, la lucha contra estas barreras se proyecta principalmente en tres grandes ámbitos: sanitario, educativo y social. Se trata así de medidas que tienen como fin el que estas personas adquieran su máximo nivel de desarrollo y autonomía personal, logren y mantengan su máxima independencia, capacidad física, sensorial, mental y social, y su inclusión y participación plena en todos los ámbitos de la vida ${ }^{16}$.

\section{3.- BIBLIOGRAFÍA}

Bariffi, F., "Capacidad jurídica y capacidad de obrar de las personas con discapacidad a la luz de la Convención de la ONU", en Pérez Bueno, L.C. (Dir.), Hacia un Derecho de la Discapacidad. Estudios en homenaje al Profesor Rafael de Lorenzo, Thomson Reuters Aranzadi, Pamplona, 2009.

Barranco Avilés, M.C y De Asís, R., "El derecho a la promoción de la autonomía personal y atención a las personas en situación de dependencia", en Barranco, M.C. (coord.), Situaciones de dependencia, Discapacidad y Derechos, Dykinson, Madrid 2011.

Cuenca, P., "La capacidad jurídica de las personas con discapacidad: el art. 12 de la Convención de la ONU y su impacto en el Ordenamiento jurídico español", en Derechos y Libertades, n. 24, 2011.

De Asís, R., "El segundo informe sobre cumplimiento por parte de España de la Convención sobre los derechos de las personas con discapacidad", en Papeles El Tiempo de los Derechos, n. 16, 2019.

\footnotetext{
15 Vid. De Asís, R., Vicente, I. y Carvalho, A., "Sobre la atención temprana como una cuestión de derechos humanos", en Actas de Cooperación Sociosanitaria, 24, 2019, Madrid, pp. 49 a 71.

16 Dentro del Derecho Internacional de los derechos humanos, existen dos grandes textos normativos que, de alguna manera, sirven para justificar este derecho: la Convención de los Derechos del Niño y la Convención sobre los derechos de las personas con discapacidad. Así, en la Convención sobre los derechos del niño son de destacar los artículos 23 y 24. Por su parte, en la Convención sobre los derechos de las personas con discapacidad, destacan en este punto los artículos 7, 24, 25 y 26.
} 
De Asís, R., Vicente, I. y Carvalho, A., "Sobre la atención temprana como una cuestión de derechos humanos", en Actas de Cooperación Sociosanitaria, 24, 2019, Madrid.

De Asís, R., "Discapacidad y Constitución", en Derechos y Libertades, n. 29, 2013.

De Asís, R., "Sobre el derecho al conocimiento y aprendizaje de la lengua de signos", en Cuenca, P. (ed.), Estudios sobre los derechos de las personas sordas, Dykinson, Madrid 2012.

Kalbag, L., Accessibility for Everyone, A Book Apart, New York 2017.

Palacios, A., El modelo social de la discapacidad, Colección CERMI, Madrid 2008.

Rodríguez-Piñeiro, M., "Artículo 49", en Rodríguez-Piñeiro, M. y Casas, M.E., Comentarios a la Constitución española, BOE-Wolters Kluwer, Madrid 2018. 